\title{
Near-field radiative heat transfer between closely spaced graphene and amorphous $\mathrm{SiO}_{2}$
}

\author{
A. I. Volokitin ${ }^{1,2, *}$ and B. N. J. Persson ${ }^{1}$ \\ ${ }^{1}$ Peter Grünberg Institut, Forschungszentrum Jülich, D-52425, Germany \\ ${ }^{2}$ Samara State Technical University, 443100 Samara, Russia \\ (Received 10 February 2011; published 27 June 2011)
}

\begin{abstract}
We study the near-field radiative energy transfer between graphene and an amorphous $\mathrm{SiO}_{2}$ substrate. In comparison with the existing theories of near-field radiative heat transfer our theory takes into account that the free carriers in graphene are moving relative to the substrate with a drift velocity $v$. In this case the heat flux is determined by both thermal and quantum fluctuations. We find that quantum fluctuations give an important contribution to the radiative energy transfer for low temperatures and high electric field (large drift velocities). For nonsuspended graphene the near-field radiative energy transfer gives a significant contribution to the heat transfer in addition to the contribution from phononic coupling. For suspended graphene (large separation) the corresponding radiative energy transfer coefficient at a nanoscale gap is $\sim 3$ orders of magnitude larger than radiative heat transfer coefficient of the blackbody radiation limit.
\end{abstract}

DOI: 10.1103/PhysRevB.83.241407

PACS number(s): 73.23.-b, 44.40.+a

Transfer of energy between two surfaces separated by a vacuum gap is a topic that has fascinated several generations of researches. If both surfaces are at rest, then at large separation $d \gg \lambda_{T}=k_{B} T / \hbar$ the radiative heat transfer is determined by the Stefan-Boltzmann law, according to which the thermal heat transfer coefficient $\alpha=4 \sigma T^{3}$. In this limiting case the heat transfer between two bodies is determined by the propagating electromagnetic waves radiated by the bodies and does not depend on the separation $d$. At $T=300 \mathrm{~K}$ this law predicts the (very small) heat transfer coefficient $\alpha \approx 6 \mathrm{~W} \mathrm{~m}^{-2} \mathrm{~K}^{-1}$. However, as was first predicted theoretically by Polder and Van Hove ${ }^{1}$ in the framework of stochastic electrodynamics introduced by Rytov $^{2-4}$ and as was recently confirmed experimentally, ${ }^{5,6}$ at short separation $d \ll \lambda_{T}$, the heat transfer may increase by many orders of magnitude due to the evanescent electromagnetic waves; this is often referred to as photon tunneling. Particularly strong enhancement occurs if the surfaces of the bodies can support localized surface modes such as surface plasmonpolaritons, surface phonon-polaritons, or adsorbate vibrational modes. $^{7,8}$

The theory of the radiative heat transfer developed in Ref. 1 is only valid for bodies at rest. A more general theory of the radiative energy transfer between moving bodies, with arbitrary relative velocities, was developed by us in Ref. 9. According to this theory, there is transfer of energy between moving bodies even at zero temperature difference, and the heat is generated by the relative motion of quantum and thermal fluctuations. It appear in its most elementary form when the surfaces are at zero Kelvin and the heat is generated by the relative movement of quantum fluctuations. In this Rapid Communication this theory is applied to calculate the radiative energy transfer between carriers (moving with the drift velocity $v$ ) in graphene and the substrate.

Graphene, the recently isolated single-layer carbon sheet, consists of carbon atoms closely packed in a flat twodimensional crystal lattice. The unique electronic and mechanical properties of graphene ${ }^{10,11}$ are being actively explored both theoretically and experimentally because of its importance for fundamental physics and for possible technological applications. ${ }^{12}$ In particular, a great deal of attention has been devoted to the applications of graphene for electronics and sensors. ${ }^{10,12}$

For nonsuspended graphene direct phononic coupling also contributes to heat transfer. ${ }^{13-16}$ Graphene interact very weakly with most substrates mainly via van der Waals forces. According to theoretical calculations, ${ }^{14-16}$ the heat transfer coefficient due to the direct phononic coupling for the interface between graphene and a perfectly smooth (amorphous) $\mathrm{SiO}_{2}$ substrate is $\alpha_{\mathrm{ph}} \approx 3 \times 10^{8} \mathrm{~W} \mathrm{~m}^{-2} \mathrm{~K}^{-1}$, and according to experiment $^{17}$ (at room temperature), the heat transfer coefficient ranges from $8 \times 10^{7}$ to $1.7 \times 10^{8} \mathrm{~W} \mathrm{~m}^{-2} \mathrm{~K}^{-1}$ (however, these values are probably influenced by the substrate surface roughness).

In this Rapid Communication we investigate heat generation and dissipation due to friction produced by the interaction between moving (drift velocity $v$ ) charge carriers in graphene and the optical phonons in nearby amorphous $\mathrm{SiO}_{2}$ and the acoustic phonons in graphene. Friction produces work and thermal heating of the graphene, which results in near-field radiative energy transfer and phononic heat transfer between the graphene and $\mathrm{SiO}_{2}$. A self-consistent theory that describes these phenomena was formulated by us in Ref. 18, and it allows us to predict experimentally measurable effects. In comparison with the existing microscopic theories of energy transfer and transport in graphene $\mathrm{e}^{19,20}$ our theory is macroscopic. The electromagnetic interaction between graphene and a substrate is described by the dielectric functions of the materials, which can be accurately determined from theory and experiment.

Consider graphene and a substrate, with flat parallel surfaces at separation $d \ll \lambda_{T}=c \hbar / k_{B} T$. Assume that the free charge carriers in graphene move with the velocity $v \ll c$ ( $c$ is the light velocity) relative to the substrate. According to Ref. 9, the frictional stress $F_{x}$ acting on the charge carriers in graphene and the radiative energy flux $S_{z}$ across the substrate surface, both mediated by a fluctuating electromagnetic field, 
are determined by

$$
\begin{aligned}
F_{x}= & \frac{\hbar}{\pi^{3}} \int_{0}^{\infty} d q_{y} \int_{0}^{\infty} d q_{x} q_{x} e^{-2 q d}\left\{\int _ { 0 } ^ { \infty } d \omega \left(\frac{\operatorname{Im} R_{d}(\omega) \operatorname{Im} R_{g}\left(\omega^{+}\right)}{\left|1-e^{-2 q d} R_{d}(\omega) R_{g}\left(\omega^{+}\right)\right|^{2}}\left[n_{d}(\omega)-n_{g}\left(\omega^{+}\right)\right]\right.\right. \\
& \left.\left.+\frac{\operatorname{Im} R_{d}\left(\omega^{+}\right) \operatorname{Im} R_{g}(\omega)}{\left|1-e^{-2 q d} R_{d}\left(\omega^{+}\right) R_{g}(\omega)\right|^{2}}\left[n_{g}(\omega)-n_{d}\left(\omega^{+}\right)\right]\right)+\int_{0}^{q_{x} v} d \omega \frac{\operatorname{Im} R_{d}(\omega) \operatorname{Im} R_{g}\left(\omega^{-}\right)}{\left|1-e^{-2 q d} R_{d}(\omega) R_{g}\left(\omega^{-}\right)\right|^{2}}\left[n_{g}\left(\omega^{-}\right)-n_{d}(\omega)\right]\right\}, \\
S_{z}= & \frac{\hbar}{\pi^{3}} \int_{0}^{\infty} d q_{y} \int_{0}^{\infty} d q_{x} e^{-2 q d}\left\{\int_{0}^{\infty} d \omega\left(-\frac{\omega \operatorname{Im} R_{d}(\omega) \operatorname{Im} R_{g}\left(\omega^{+}\right)}{\left|1-e^{-2 q d} R_{d}(\omega) R_{g}\left(\omega^{+}\right)\right|^{2}}\left[n_{d}(\omega)-n_{g}(\omega)^{+}\right)\right]\right. \\
& \left.\left.+\frac{\omega^{+} \operatorname{Im} R_{d}\left(\omega^{+}\right) \operatorname{Im} R_{g}(\omega)}{\left|1-e^{-2 q d} R_{d}\left(\omega^{+}\right) R_{g}(\omega)\right|^{2}}\left[n_{g}(\omega)-n_{d}\left(\omega^{+}\right)\right]\right)+\int_{0}^{q_{x} v} d \omega \frac{\omega \operatorname{Im} R_{d}(\omega) \operatorname{Im} R_{g}\left(\omega^{-}\right)}{\left|1-e^{-2 q d} R_{d}(\omega) R_{g}\left(\omega^{-}\right)\right|^{2}}\left[n_{g}\left(\omega^{-}\right)-n_{d}(\omega)\right]\right\},
\end{aligned}
$$

where $n_{i}(\omega)=\left[\exp \left(\hbar \omega / k_{B} T_{i}\right)-1\right]^{-1}(i=g, d), T_{g(d)}$ is the temperature of graphene (substrate), $R_{i}$ is the reflection amplitude for surface $i$ for $p$-polarized electromagnetic waves, and $\omega^{ \pm}=\omega \pm q_{x} v$. The reflection amplitude for graphene (substrate) for $q \gg \sqrt{\left|\epsilon_{d}(\omega)\right|} \omega / c$ is determined by ${ }^{21}$

$$
R_{g(d)}=\frac{\epsilon_{g(d)}-1}{\epsilon_{g(d)}+1},
$$

where $\epsilon_{g(d)}$ is the dielectric function for graphene (substrate). In the study below we used the dielectric function of graphene, which was calculated recently within the random-phase approximation (RPA). ${ }^{22,23}$ The different pieces of this dielectric function on a real axis in the complex plane can be obtained from a complex-valued function that is analytical in the upper half-plane of the complex $\omega$ plane. ${ }^{18}$ The dielectric function of amorphous $\mathrm{SiO}_{2}$ can be described using an oscillator model. ${ }^{24}$

According to Eqs. (1) and (2) in the case when free carriers are moving relative to the substrate both thermal and quantum fluctuations give contributions to the frictional stress and the radiative energy transfer. This situation is different from that considered in Refs. 14 and 15, where it was assumed that the free carriers in graphene had vanishing drift velocity. The contribution of the quantum fluctuations to the frictional stress was investigated by us in Ref. 18. According to Eq. (2), the contribution to the near-field energy transfer from quantum fluctuations is determined by

$$
\begin{aligned}
S_{z}^{\text {quant }}= & S_{z}\left(T_{d}=T_{g}=0\right)=-\frac{\hbar}{\pi^{3}} \int_{0}^{\infty} d q_{y} \int_{0}^{\infty} d q_{x} \\
& \times \int_{0}^{q_{x} v} d \omega \omega e^{-2 q d} \frac{\operatorname{Im} R_{d}(\omega) \operatorname{Im} R_{g}\left(\omega^{-}\right)}{\left|1-e^{-2 q d} R_{d}(\omega) R_{g}\left(\omega^{-}\right)\right|^{2}} .
\end{aligned}
$$

The steady-state temperature can be obtained from the condition that the power generated by friction must be equal to the energy transfer across the substrate surface,

$$
F_{t}\left(T_{d}, T_{g}\right) v=S_{z}\left(T_{d}, T_{g}\right)+\alpha_{\mathrm{ph}}\left(T_{g}-T_{d}\right),
$$

where $F_{t}$ is the total friction force, which is the sum of the extrinsic friction force $F_{x}$, due to interaction with optical phonons in $\mathrm{SiO}_{2}$, and the intrinsic friction force due interaction with acoustic and optical phonons in graphene. The friction force due to interaction with acoustic phonons at low velocities is determined by $F_{a c}=n e \mu^{-1} v$, where $\mu$ is low-field mobility due to scattering of the carriers against the acoustic phonons of graphene. At room temperature $\mu \approx 20 \mathrm{~m}^{-2} \mathrm{~V}^{-1} \mathrm{~s}^{-1} \cdot{ }^{25} \mathrm{At}$ other temperatures the mobility can be obtained taking into account that $\mu^{-1}$ depends approximately linearly on $T_{g}$. The friction force acting on the charge carriers in graphene for high electric field (large velocities) is determined by the interaction with the optical phonons of the graphene and with the optical phonons of the substrate. The frequency of optical phonons in graphene is a factor 4 larger than for the optical phonon in $\mathrm{SiO}_{2}$. Thus, one can expect that for graphene on $\mathrm{SiO}_{2}$ the high-field friction force will be determined by excitations of optical phonons in $\mathrm{SiO}_{2}$. The second term on the right side of Eq. (5) takes into account the heat transfer through direct phononic coupling; $\alpha_{\mathrm{ph}}$ is the heat transfer coefficient due to phononic coupling. Due to weakness of the van der Waals interaction between graphene and the substrate the application of the theory outlined above is justified for both suspended and nonsuspended graphene.

As discussed above, for graphene on $\mathrm{SiO}_{2}$ the excess heat generated by the current is transferred to the substrate through the near-field radiative heat transfer and via the direct phononic coupling (for which the heat transfer coefficient $\left.\alpha \approx 10^{8} \mathrm{~W} \mathrm{~m} \mathrm{~m}^{-2} \mathrm{~K}^{-1}\right)$. At small temperature differences ( $\Delta T=T_{g}-T_{d} \ll T_{d}$ ), from Eq. (5) we get

$$
\Delta T=\frac{F_{t 0} v-S_{z 0}}{\alpha_{\mathrm{ph}}+S_{z 0}^{\prime}-F_{t 0}^{\prime} v},
$$

where $F_{t 0}=F_{t}\left(T_{d}, T_{g}=T_{d}\right), S_{z 0}=S_{z}\left(T_{d}, T_{g}=T_{d}\right)$,

$$
F_{t 0}^{\prime}=\left.\frac{d F_{t}\left(T_{d}, T_{g}\right)}{d T_{g}}\right|_{T_{g}=T_{d}}, \quad S_{z 0}^{\prime}=\left.\frac{d S_{z}\left(T_{d}, T_{g}\right)}{d T_{g}}\right|_{T_{g}=T_{d}} .
$$

We note that, in contrast to the heat transfer between bodies at rest, for moving bodies the energy flux $S_{z}\left(T_{d}, T_{g}\right)$ is not equal to zero even for the case when there is no temperature 
difference between the bodies. The energy transfer coefficient is given by

$$
\alpha=\frac{S_{z}\left(T_{d}, T_{g}\right)+\alpha_{\mathrm{ph}} \Delta T}{\Delta T} \approx \frac{\left(\alpha_{\mathrm{ph}}+S_{z 0}^{\prime}\right) F_{t 0} v-S_{z 0} F_{t 0}^{\prime} v}{F_{t 0} v-S_{z 0}} .
$$

For small velocities $F_{t 0} \sim v$ and $S_{z 0} \sim v^{2}$. Thus from Eq. (7) it follows that in the limit $v \rightarrow 0$ the energy transfer coefficient between moving bodies is not reduced to the heat transfer coefficient between bodies at rest, which is determined by $\alpha_{t h}=\alpha_{\mathrm{ph}}+S_{z 0}^{\prime}$. This effect is due to the term $S_{z 0}$ in the total energy flux, which exists only between moving bodies. The energy transfer coefficient can be strongly enhanced in comparison to the heat transfer coefficient when $F_{t 0} v \approx S_{z 0}$. Figure 1(a) shows the ratio of the energy transfer coefficient to the phononic heat transfer coefficient for $d=0.35 \mathrm{~nm}$ and $n=10^{16} \mathrm{~m}^{-2}$. For low and intermediate field this ratio is larger than unity, which means that in this region the near-field radiative energy transfer gives additional significant contribution to the heat transfer due to direct phononic coupling. For nonsuspended graphene on $\mathrm{SiO}_{2}$ the energy transfer is very effective, and the temperature difference does not rise high, even for such high electric field that saturation in the $I-E$ characteristic starts ${ }^{26}$ [see Fig. 1(b)]. The radiative heat transfer between bodies at rest is determined only by thermal fluctuations, in contrast to the radiative energy transfer between moving bodies, which is determined by both thermal and quantum fluctuations. Figure 1(c) shows that quantum fluctuations can give significant contribution to the total energy transfer for low temperatures and large electric field (high drift velocity). Similarly, in the (electric current) saturation region quantum fluctuations give significant contribution to the
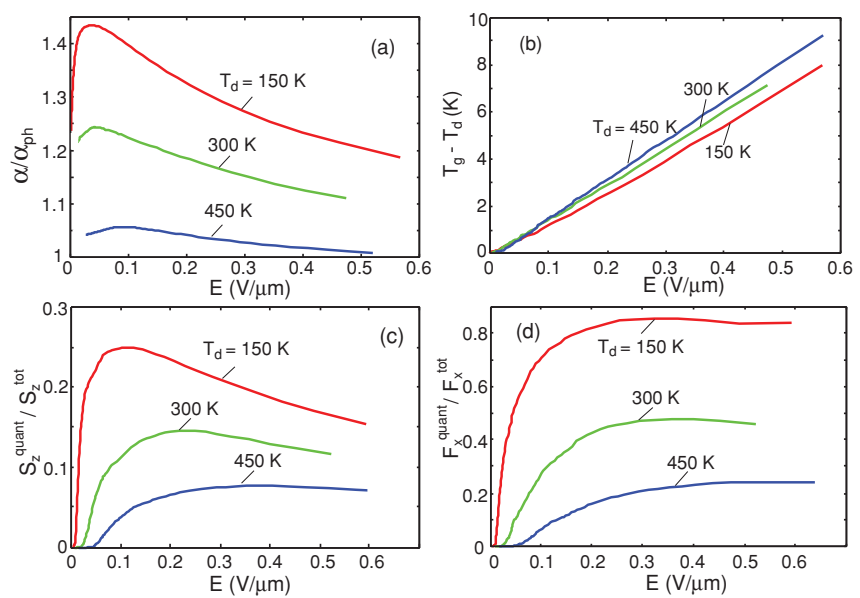

FIG. 1. (Color online) Radiative energy transfer between graphene and $\mathrm{SiO}_{2}$ for $n=10^{16} \mathrm{~m}^{-2}, d=0.35 \mathrm{~nm}$, and $\alpha_{\mathrm{ph}}=$ $1.0 \times 10^{8} \mathrm{~W} \mathrm{~m}^{-2} \mathrm{~K}^{-1}$. (a) The dependence of the ratio between the total energy transfer coefficient and the phononic heat transfer coefficient on electric field. (b) Dependence of the temperature difference between graphene and substrate on the electric field. (c) Dependence of the ratio between the heat flux only due to quantum fluctuations $S_{z}^{\text {quant }}$ and the total energy flux on the electric field. (d) Dependence of the ratio between the friction force only due to quantum fluctuations $F_{x}^{\text {quant }}$ and the total friction force on the electric field.
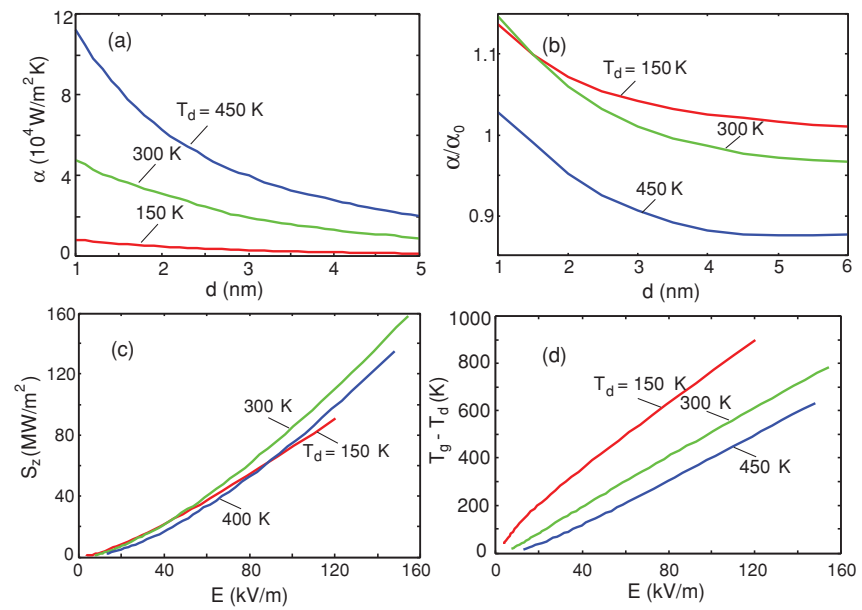

FIG. 2. (Color online) Radiative energy transfer between graphene and $\mathrm{SiO}_{2}$ for $n=10^{12} \mathrm{~cm}^{-2}$ and $\alpha_{\mathrm{ph}}=0$. (a) Dependence of the energy transfer coefficient on the separation $d$ for low electric field $(v \rightarrow 0)$. (b) Dependence of the ratio between the energy transfer coefficient and the heat transfer coefficient on the separation $d$ for low electric field $(v \rightarrow 0)$. (c) Dependence of the radiative energy flux on electric field for $d=1.0 \mathrm{~nm}$. (d) Dependence of the temperature difference between graphene and substrate on electric field for $d=1.0 \mathrm{~nm}$.

total friction force, which is determined, as discussed above, by the sum of the extrinsic and intrinsic friction forces [see Fig. 1(d)]. The extrinsic friction force has contributions from both thermal and quantum fluctuations. The friction force due to quantum fluctuations is usually called quantum friction, which was discussed by us recently in Ref. 18 .

Figure 2(a) shows the dependence of the energy transfer coefficient on the separation $d$ for low electric field $(v \rightarrow 0)$. At $d \sim 5 \mathrm{~nm}$ and $T=300 \mathrm{~K}$ the energy transfer coefficient, due to the near-field radiative energy transfer, is $\sim 10^{4} \mathrm{~W} \mathrm{~m}^{-2} \mathrm{~K}^{-1}$, which is $\sim 3$ orders of magnitude larger than the radiative heat transfer coefficient of the blackbody radiation. In comparison, the near-field radiative heat transfer coefficient in a $\mathrm{SiO}_{2}$ $\mathrm{SiO}_{2}$ system for the plate-plate configuration, when extracted from experimental data ${ }^{5}$ for the plate-sphere configuration, is $\sim 2230 \mathrm{~W} \mathrm{~m}^{-2} \mathrm{~K}^{-1}$ at an $\sim 30 \mathrm{~nm}$ gap. For this system the radiative heat transfer coefficient depends on the separation as $1 / d^{2}$. Thus $\alpha \sim 10^{5} \mathrm{~W} \mathrm{~m}^{-2} \mathrm{~K}^{-1}$ at $d \sim 5 \mathrm{~nm}$, which is 1 order of magnitude larger than for the graphene- $\mathrm{SiO}_{2}$ system in the same configuration. However, the sphere has a characteristic roughness of $\sim 40 \mathrm{~nm}$, and the experiments ${ }^{5,6}$ were restricted to separation wider than $30 \mathrm{~nm}$ (at smaller separation the imperfections affect the measured heat transfer). Thus the extreme near-field separation, with $d$ less than approximately $10 \mathrm{~nm}$, may not be accessible using a plate-sphere geometry. A suspended graphene sheet has a roughness of $\sim 1 \mathrm{~nm},{ }^{27}$ and measurements of the thermal contact conductance can be performed from separation larger than $\sim 1 \mathrm{~nm}$. At such separation one would expect the emergence of nonlocal and nonlinear effects. This range is of great interest for the design of nanoscale devices, as modern nanostructures are considerably smaller than $10 \mathrm{~nm}$ and are separated in some cases by only a few Angstroms. 
Figure 2(b) shows that at small separation there is significant difference between the radiative energy transfer coefficient and the radiative heat transfer coefficient determined (in absence of direct phononic coupling) by $\alpha_{0}=S_{z 0}^{\prime}$. This difference vanishes for large separation because $S_{z 0}$ and $F_{x 0}$ rapidly decrease when separation increases. At large separation the friction force is dominated by the intrinsic friction and in this case $\alpha \approx \alpha_{0}$. Figure 2(c) shows the dependence of the radiative energy flux on electric field for $d=1 \mathrm{~nm}$. For this separation the energy transfer is considerably less effective than for $d=0.35 \mathrm{~nm}$, which leads to a rapid increase of the temperature difference [see Fig. 2(d)]. High temperatures are achieved at low electric field (small drift velocities) when the contribution to the radiative energy transfer from quantum fluctuations is very small and the energy transfer is mainly determined by thermal fluctuations.

In conclusion, we have used theories of the van der Waals friction and the near-field radiative energy transfer to study heat generation and dissipation in graphene due to the interaction with phonon-polaritons in the (amorphous) $\mathrm{SiO}_{2}$ substrate and acoustic phonons in graphene. For the low-field energy transfer between nonsuspended graphene and the substrate, radiative energy transfer gives a significant contribution in addition to the phononic heat transfer. High-field heat transfer is determined by the phononic mechanism. For high electric field (large drift velocities) and low temperatures quantum fluctuations give an important contribution to the energy flux and the friction force. For suspended graphene the energy transfer coefficient at a nanoscale gap is $\sim 3$ orders of magnitude larger than the radiative heat transfer coefficient of the blackbody radiation limit. We have pointed out that graphene can be used to study near-field radiative heat transfer in the plate-plate configuration and for shorter separations than is possible now in the plate-sphere configuration.

A.I.V acknowledges financial support from the Russian Foundation for Basic Research (Grant No. N 10-02-00297-à) and ESF within the project "New Trends and Applications of the Casimir Effect." *alevolokitin@yandex.ru

${ }^{1}$ D. Polder and M. Van Hove, Phys. Rev. B 4, 3303 (1971).

${ }^{2}$ S. M. Rytov, Theory of Electrical Fluctuation and Thermal Radiation (Academy of Science of USSR Publishing, Moscow, 1953).

${ }^{3}$ M. L. Levin and S. M. Rytov, Theory of Eqilibrium Thermal Fluctuations in Electrodynamics (Science Publishing, Moscow, 1967).

${ }^{4}$ S. M. Rytov, Y. A. Kravtsov, and V. I. Tatarskii, Principles of Statistical Radiophyics (Springer, New York, 1989), Vol. 3.

${ }^{5}$ S. Shen, A. Narayanaswamy, and G. Chen, Nano Lett. 9, 2909 (2009).

${ }^{6}$ E. Rousseau, A. Siria, G. Jourdan, S. Volz, F. Comin, J. Chevrier, and J. J. Greffet, Nat. Photon. 3, 514 (2009).

${ }^{7}$ K. Joulain, J. P. Mulet, F. Marquier, R. Carminati, and J. J. Greffet, Surf. Sci. Rep. 57, 59 (2005).

${ }^{8}$ A. I. Volokitin and B. N. J. Persson, Rev. Mod. Phys. 79, 1291 (2007).

${ }^{9}$ A. I. Volokitin and B. N. J. Persson, Phys. Rev. B 78, 155437 (2008).

${ }^{10}$ K. S. Novoselov, A. K. Geim, S. V. Morosov, D. Jiang, Y. Zhang, S. V. Dubonos, I. V. Grigorieva, and A. A. Firsov, Science 306, 666 (2004).

${ }^{11}$ K. S. Novoselov, S. V. Morosov, M. I. Katsnelson, I. V. Grigorieva, S. V. Dubonos, and A. A. Firsov, Nature (London) 197, 197 (2005).

${ }^{12}$ A. K. Geim and K. S. Novoselov, Nat. Mater. 6, 183 (2007).
${ }^{13}$ Z. Y. Ong and E. Pop, e-print arXiv:1101.2463v1 (to be published).

${ }^{14}$ B. N. J. Persson and H. Ueba, Europhys. Lett. 91, 56001 (2010).

${ }^{15}$ B. N. J. Persson and H. Ueba, J. Phys. Condens. Matter 22, 462201 (2010).

${ }^{16}$ B. N. J. Persson, A. I. Volokitin, and H. Ueba, J. Phys. Condens. Matter 23, 045009 (2011).

${ }^{17}$ Z. Chen, W. Jang, W. Bao, J. D. Lau, C. N. Chen, and C. Dames, Appl. Phys. Lett. 95, 161910 (2009).

${ }^{18}$ A. I. Volokitin and B. N. J. Persson, Phys. Rev. Lett. 106, 094502 (2011).

${ }^{19}$ S. V. Rotkin, V. Perebeinos, A. G. Petrov, and P. Avouris, Nano Lett. 9, 1850 (2009)

${ }^{20}$ V. Perebeinos and P. Avouris, Phys. Rev. B 81, 195442 (2010).

${ }^{21}$ A. I. Volokitin and B. N. J. Persson, J. Phys. Condens. Matter 13, 859 (2001).

${ }^{22}$ B. Wunscvh, T. Stauber, F. Sols, and F. Guinea, New J. Phys. 8, 318 (2006).

${ }^{23}$ E. H. Hwang and S. Das Sarma, Phys. Rev. B 75, 205418 (2007).

${ }^{24}$ D. Z. A. Chen, R. Hamam, M. Soljačić, and J. D. Joannopoulos, Appl. Phys. Lett. 90, 181921 (2007).

${ }^{25}$ J. H. Chen, C. Jang, S. Xiao, M. Ishigami, and M. S. Fuhrer, Nat. Nanotechnol. 3, 206 (2008).

${ }^{26}$ M. Freitag, M. Steiner, Y. Martin, V. Perebeinos, Z. Chen, J. C. Tsang, and P. Avouris, Nano Lett. 9, 1883 (2009).

${ }^{27}$ J. C. Meyer, A. K. Geim, M. I. Katsnelson, K. S. Novoselov, T. J. Booth, and S. Roth, Nature (London) 446, 60 (2007). 